\title{
THE TOMITA DECOMPOSITION OF RINGS OF OPERATORS
}

BY

JOSEPH L. TAYLOR(1)

I. Introduction. It is known that if $R$ is a symmetric ring of bounded operators on a separable Hilbert space $H$ and $\xi_{0}$ is a vector in $H$ which is cyclic with respect to $R$, then the positive functional $F(A)=\left(A \xi_{0}, \xi_{0}\right)$, for $A \in R$, may be written as a direct integral over a compact Hausdorff space $M$, i.e., $F(A)=\int_{M} f_{m}(A) d \mu(m)$ where $\mu$ is a positive regular Borel measure and the functionals $f_{m}$ are indecomposable except, at most, for $m \in M_{0} \subset M$ and $\mu\left(M_{0}\right)=0$. This decomposition of $F$ induces a representation of $R$ as a direct integral of rings $R_{m}$ of operators on a Hilbert space $H_{m}$ and for almost all $m(\bmod \mu), R_{m}$ is an irreducible ring on $H_{m}$.

The problem of extending this type of decomposition to rings of operators on an arbitrary Hilbert space was attacked in 1954 by Tomita (cf. [6]) using extremely penetrating techniques. However, certain parts of Tomita's development of his decomposition theory require a special measure theoretic result which is not valid in general. Consequently, the question of whether or not this measure theoretic difficulty could be circumvented arose; i.e., did the Tomita decomposition hold for arbitrary rings and, if not, for what type of rings did it hold?

In this paper we shall first show (Theorem 2.3) that in case $R$ is a weakly closed symmetric ring which contains its commutant, then the Tomita decomposition holds and, in fact, all of the rings $R_{m}$ are irreducible; furthermore $R$ is completely determined by the representation of $H$ as a direct integral. In $\$ 3$ of this paper we shall construct a symmetric ring $R$ where the Tomita decomposition fails to hold (Theorem 3.3). In our example no one of the rings $R_{m}$ is irreducible. We conclude the paper in $\S 4$ with some remarks indicating how recent results of Loomis [4] can be applied to obtain irreducible decomposition of rings of operators.

II. Notation and terminology. Throughout this paper we shall use the notation and terminology employed in Naimark's treatise, [5]. Also, we shall use the ring theory developed in [5] together with those parts of Tomita's formulation of the decomposition problem which are valid.

Let $H$ denote an arbitrary Hilbert space, $R_{1}$ a symmetric Banach ring of operators on $H$, and $E$ a maximal commutative subring contained in the commutant of $R_{1}$. Let $R=R_{1} \cup E$; hence, $E$ is both the center and the commutant of $R$. We

Presented to the Society, August 30, 1963; received by the editors May 20, 1963.

(1) This research was supported in part by a National Science Foundation grant. 
shall denote the maximal ideal space of $E$ by $M$ and the continuous functions on $M$ by $C(M) . N$ will denote the set of normalized indecomposable positive functionals on $R$ and $\bar{N}$ the closure of $N$ in the weak-* topology of $R^{*}$, where $R^{*}$ is the dual of $R$. Finally, we shall assume that $H$ contains a vector $h_{0}$ of norm one such that $h_{0}$ is cyclic with respect to $R$. We set $F(A)=\left(A h_{0}, h_{0}\right), A \in R$, so that $F \in R^{*}$. Theorem 2.1 and 2.2 below summarize certain parts of Tomita's theory that we shall need; for the details of these theorems, the reader is referred to [5].

THEOREM 2.1. There exists a homeomorphism, denoted by $m \rightarrow f_{m}$, of $M$ into $\bar{N}$ and a positive, normalized regular Borel measure $\mu$ on $M$ such that

(1) $F(A)=\int_{M} f_{m}(A) d \mu(m)$, for each $A \in R$,

(2) $f_{m}(A B)=f_{m}(A) x_{B}(m)$, for each $A \in R$ and each $B \in E$, where $B \rightarrow x_{B}(m)$ is the natural isomorphism of $E$ onto $C(M)$,

(3) the mapping $m \rightarrow f_{m}$ and the measure $\mu$ are uniquely determined by (1) and (2), and

(4) the carrier of $\mu$ is all of $M$.

For the verification of these statements see [5], in particular, II, p. 507; Theorem 1, p. 493, and III, p. 507.

Before stating Theorem 2.2, we shall use the above theorem to formulate the notion of a direct integral Hilbert space. This is done in the following manner. For each $m \in M$, the functional $f_{m}$ determines a Hilbert space $H_{m}$, a ring of operators $R_{m}$ on $H_{m}$, and a representation $A \rightarrow A_{m}$ of the ring $R$ onto the ring $R_{m}$. There exists a vector $\xi_{0}(m) \in H_{m}$ of norm one such that $\xi_{0}(m)$ is cyclic with respect to $R_{m}$ and $f_{m}(A)=\left(A_{m} \xi_{0}(m), \xi_{0}(m)\right)$ for all $A \in R$. The set $S$ of all vector valued functions of the form $\eta(m)=A_{m} \xi_{0}(m), A \in R$, is a Euclidean space under the inner product

$$
(\xi, \eta)=\int_{M}(\xi(m), \eta(m)) d \mu(m)=\int_{M} f_{m}\left(A^{*} C\right) d \mu(m),
$$

where $A, C \in R, \xi(m)=C_{m} \xi_{0}(m), A^{*}$ is the adjoint of $A$. The completion of $S$, denoted by $\hat{H}$, is a Hilbert space and is called the direct integral of the spaces $H_{m}$ with respect to $\mu . S$ is called the basis of the direct integral space $\hat{H}$.

THEOREM 2.2. There exists a unitary operator $U$ mapping $H$ onto $\hat{H}$ and an isometric mapping $A \rightarrow \hat{A}$ of $R$ into $B(\hat{H})$ such that

(1) $A=U^{-1} \hat{A} U$, for $A \in R$,

(2) $\hat{A} S \subset S$, for $A \in R$,

(3) $(\hat{A} \xi)(m)=A_{m} \xi(m)$, for $A \in R, \xi \in S, m \in M$, and

(4) if $B \in E$, then $(\hat{B} \xi)(m)=x_{B}(m) \xi(m)$ for $\xi \in S$ and $m \in M$; moreover, every continuous function on $M$ defines an operator $B$ in this fashion.

We refer the reader to pp. 512-515 in [5]. 
The image $\hat{R}$ under the mapping $A \rightarrow \hat{A}$ is called a direct integral of the rings $R_{m}$. (3) shows that each operator $\hat{A}$ may be considered as an operator valued function on $M$ with values in the rings $R_{m}$. The essential problem in this type of decomposition is to determine if almost all of the rings $R_{m}$ are irreducible; i.e., $R_{m}$ is irreducible except for $m \in M_{0}$ where $\mu\left(M_{0}\right)=0$. We shall now show that this is the case when $R$ is weakly closed. We note that if $R$ is weakly closed then $R$ is the commutant of $E$.

We shall need two lemmas. In these lemmas we continue to use the notations and facts cited above.

LEMMA 2.1. If $y$ is an essentially bounded $\mu$ measurable function on $M$, then there exists $B \in E$ such that $y(m)=x_{B}(m)$ almost everywhere, where $B \rightarrow x_{B}(m)$ is in accordance with (2) of Theorem 2.1 .

Proof. Suppose $y \in L_{\infty}(\mu)$ and $0 \leqq y(m) \leqq 1$ for almost all $m \in M$. We define $G(A)=\int_{M} f_{m}(A) y(m) d \mu(m)$ for $A \in R$; hence, $G$ is a positive functional on $R$ and $G(A) \leqq F(A)=\left(A h_{0}, h_{0}\right)$, whenever $A$ is a positive definite operator. Under these circumstances, there exists $B \in E$ such that $G(A)=F(A B)$ for $A \in R$ (cf. Theorem 1, p. 262 of [5]). Hence $\int_{M} f_{m}(A) y(m) d \mu(m)=\int_{M} f_{m}(A) x_{B}(m) d \mu(m)$ for $A \in R$.

In particular, the equality holds if we replace $A$ by $C, C \in E$, and, using $f_{m}(C)=f_{m}(I C)=f_{m}(I) x_{C}(m)=x_{C}(m) \quad((2)$ of Theorem 2.1), we have that $\int_{M} k(m) y(m) d \mu(m)=\int_{M} k(m) x_{B}(m) d \mu(m)$ for every continuous function $k$ on $M$. Hence, $x_{B}(m)=y(m)$ almost everywhere. The conclusion of Lemma 2.1 now follows immediately inasmuch as any function in $L_{\infty}(\mu)$ is a linear combination of functions of the above type.

LEMMA 2.2. $M$ is extremely disconnected, i.e., the closure of any open set is open.

Proof. Suppose $V$ is an open subset of $M$ and $\chi_{V}$ the characteristic function of $V$ on $M$. In accordance with Lemma 2.1 , there exists a continuous function $x_{B}$, such that $0 \leqq x_{B} \leqq 1$, and $x_{B}=\chi_{V}$ almost everywhere. Hence, if $V_{1}=\{m \in M$; $\left.x_{B}(m)=1\right\}$ and $V_{2}=\left\{m \in M: x_{B}(m)>0\right\}$, then $V_{1}$ is a closed set and $V_{2}$ is an open set. It follows that $\mu\left(V \cap\left(M-V_{1}\right)\right)=0$ inasmuch as $x_{B}<\chi_{V}$ on $V \cap\left(M-V_{1}\right)$. However, the set $V \cap\left(M-V_{1}\right)$ is an open set and, in view of (4) of Theorem 2.1, we must have $V \cap\left(M-V_{1}\right)=\varnothing$ or, what is the same, $V \subset V_{1}$. Also, if $\bar{V}$ denotes the closure of $V$, a similar argument shows that $V_{2} \cap(M-\bar{V})=\varnothing$, or $V_{2} \subset \bar{V}$. Hence, $V \subset V_{1} \subset V_{2} \subset \bar{V}$ and, since $V_{1}$ is a closed set, $V_{1}=\bar{V}$, so that $V_{1}=V_{2}=\bar{V}$. Since $V_{2}$ is an open set, $\bar{V}$ is an open set.

THEOREM 2.3. Suppose $R$ is a symmetric ring of operator over a Hilbert space $H$ such that

(1) the center $E$ of $R$ is also the commutant of $R$,

(2) $R$ is weakly closed, and 
(3) there exists a vector $h_{0} \in H$ with norm one which is cyclic with respect to $R$ and $F(A)=\left(A h_{0}, h_{0}\right)$ for $A \in R$.

Then (a) the direct integral decomposition $F(A)=\int_{M} f_{m}(A) d \mu(m)$ has the property that each of the functionals $f_{m}$ is indecomposable, and

(b) the direct integral decomposition of $R$ given by Theorem 2.2 has the property that each of the rings $R_{m}$ is irreducible over the Hilbert space $H_{m}$.

Proof. We will show that each of the rings $R_{m}$ is irreducible and it will follow from this that each of the functionals $f_{m}$ is indecomposable, inasmuch as the ring $R_{m}$ is the image of $R$ under the representation generated by $f_{m}$. In order to show that the ring $R_{m}$ is irreducible, it is sufficient to prove that if $n \in M, \eta_{n} \in H_{n}$, $\gamma_{n} \in H_{n}, \gamma_{n} \neq 0$, and $\varepsilon>0$, then there exists $A \in R$ such that $\left|A_{n} \gamma_{n}-\eta_{n}\right|<\varepsilon$. We shall prove this first for the case where $\eta_{n}$ and $\gamma_{n}$ are of the form $\eta_{n}=C_{n} \xi_{0}(n)$ and $\gamma_{n}=D_{n} \xi_{0}(n)$ for $C \in R$ and $D \in R$.

If $\gamma_{n} \neq 0$, then, since the function $\left|D_{m} \xi_{0}(m)\right|^{2}=f\left(D_{m}^{*} D_{m}\right)$ is a continuous function on $M$, there exists an open set $V \subset M$ such that $n \in V,\left|D_{m} \xi_{0}(m)\right|$ $\geqq 2^{-1}\left|D_{n} \xi_{0}(n)\right|=\delta_{1}$, and $\left|C_{m} \xi_{0}(m)\right| \leqq\left|C_{n} \xi_{0}(n)\right|+1=\delta_{2}$, for $m \in V$. In view of Lemma 2.2 we can select $V$ so that it is compact and, hence, the characteristic function of $V$ on $M$, say $\chi_{V}$, is a continuous function and there exists a $P \in E$ such that $x_{P}=\chi_{V}$ everywhere. We note that $P^{2}=P$.

We now define the subspace $K$ of $H$ to be the closed subspace generated by vectors of the form $h_{1}=B P h_{0}$ for $B \in E$. For each such vector $h_{1}$ we have $\left|D h_{1}\right|^{2}=\int_{M}\left|D_{m} P_{m} B_{m} \xi_{0}(m)\right|^{2} d \mu(m)=\int_{M}\left|x_{P}(m) x_{B}(m)\right|^{2}\left|D_{m} \xi_{0}(m)\right|^{2} d \mu(m)$ $=\int_{V}\left|x_{B}(m)\right|^{2}\left|D_{m} \xi_{0}(m)\right|^{2} d \mu(m) \geqq \delta_{1}^{2} \int_{V}\left|x_{B}(m)\right|^{2} d \mu(m)=\delta_{1}^{2} \int_{M}\left|P_{m} B_{m} \xi_{0}(m)\right|^{2} d \mu(m)$ $=\delta_{1}^{2}\left|h_{1}\right|^{2}$. Similarly, $\left|C h_{1}\right| \leqq \delta_{2}\left|h_{1}\right|$.

Now each vector $h \in H$ can be uniquely expressed in the form $h=D h_{1}+h_{2}$ where $h_{1} \in K$ and $h_{2}$ is in the orthogonal complement of the space $D K$. Thence, if we define a linear operator $A$ on $H$ by $A h=C h_{1}$, then

$$
|A h|=\left|C h_{1}\right|=\left|C h_{1}\right|\left(\left|D h_{1}\right|\right)^{-1}\left|D h_{1}\right| \leqq \delta_{2} \delta_{1}^{-1}\left|D h_{1}+h_{2}\right|,
$$

so that $A$ is a bounded operator and $|A| \leqq \delta_{2} \delta_{1}^{-1}$. Now, if $B \in E$, then $B A h$ $=B C h_{1}=C B h_{1}$, but $B h_{1} \in K$ and $B h_{2} \perp D K$, so that $C B h_{1}=A\left(D B h_{1}+B h_{2}\right)$ $=A\left(B D h_{1}+B h_{2}\right)=A B h$. Hence, $A$ is in the commutant of $E$ and, since $R$ is weakly closed, the commutant of $E$ is $R$; i.e., $A \in R$. We also have

$$
A_{n} \gamma_{n}=A_{n} D_{n} \xi_{0}(n)=\left(\hat{A} \hat{D} \xi_{0}\right)(n)=\left(\hat{C} \xi_{0}\right)(n)=C_{n} \xi_{0}(n)=\eta_{n},
$$

which establishes the assertion we made above for vectors of the form $\eta_{n}=C_{n} \xi_{0}(n)$ and $\gamma_{n}=D_{n} \xi_{0}(n)$. Finally, we also note that

$$
|A| \leqq \delta_{2} \delta_{1}^{-1} \leqq 2\left(\left|C_{n} \xi_{0}(n)\right|+1\right)\left(\left|D_{n} \xi_{0}(n)\right|\right)^{-1} .
$$

Suppose $\alpha_{n}$ and $\beta_{n}$ are arbitrary vectors in $H_{n}, \alpha_{n} \neq 0$, and $\varepsilon>0$. Since the vectors $C_{n} \xi_{0}(n), C \in R$, are dense in $H_{n}$, there exist $C \in R$ and $D \in R$ such that 
$\left|C_{n} \xi_{0}(n)-\beta_{n}\right|<2^{-1} \varepsilon,\left|D_{n} \xi_{0}(n)-\alpha_{n}\right|<\varepsilon\left(4\left|\alpha_{n}\right|\right)^{-1}\left(\left|\beta_{n}\right|+1\right),\left|C_{n} \xi_{0}(n)\right|=\left|\beta_{n}\right|$, and $\left|D_{n} \xi_{0}(n)\right|=\left|\alpha_{n}\right|$. On the basis of the construction in the preceding paragraph there exists an operator $A \in R$ such that $A_{n} D_{n} \xi_{0}(n)=C_{n} \xi_{0}(n)$ and $|A|$ $\leqq 2\left(\left|C_{n} \xi_{0}(n)\right|+1\right)\left(\left|D_{n} \xi_{0}(n)\right|^{-1}\right)=2\left(\left|\beta_{n}\right|+1\right)\left(\left|\alpha_{n}\right|^{-1}\right)$. Hence,

$$
\begin{aligned}
\left|A_{n} \alpha_{n}-\beta_{n}\right| & \leqq\left|A_{n} D_{n} \xi_{0}(n)-A_{n} \alpha_{n}\right|+\left|A_{n} D_{n} \xi_{0}(n)-C_{n} \xi_{0}(n)\right|+\left|C_{n} \xi_{0}(n)-\beta_{n}\right| \\
& =\left|A_{n} D_{n} \xi_{0}(n)-A_{n} \alpha_{n}\right|+\left|C_{n} \xi_{0}(n)-\beta_{n}\right| \\
& \leqq|A| \cdot\left|D_{n} \xi_{0}(n)-\alpha_{n}\right|+\left|C_{n} \xi_{0}(n)-\beta_{n}\right|<\varepsilon .
\end{aligned}
$$

This completes the proof of Theorem 2.3 .

III. An example. Suppose $R_{1}$ is a symmetric Banach ring of operators on the Hilbert space $H, E$ a maximal commutative subring of the commutant of $R_{1}, R_{2}$ $=R_{1} \cup E$, and $R$ the weak closure of $R_{2}$ (i.e., $R$ is the commutant of $E$ ). Then, in accordance with Theorem 2.3, the Tomita decomposition of $R$ yields a representation of $R$ as a direct integral of irreducible rings over $M$; the maximal ideal space of $E$; moreover, $R$ is completely determined by the representation of $H$ as a direct integral over $M$, since $R$ is the commutant of $E$ and $E$ is precisely (within unitary equivalence) the ring of operators (on this direct integral space) determined by the continuous functions on $M$. The ring $R_{2}$ also has a Tomita decomposition and it is related to the decomposition of the ring $R$ in the following manner; in the representation of $R$ as a direct integral of rings $R_{m}$, the image of $R_{2}$ is exactly the Tomita representation of $R_{2}$ as a direct integral of the rings $R_{2, m} \subset R_{m}$. However, the rings $R_{2, m}$ may no longer be irreducible; furthermore, $R_{2}$ is not characterized by the representation of $H$ as a direct integral, and certainly $R_{1}$ is not characterized in this manner. We shall verify all of these statements by an example. Several lemmas and definitions will be needed in order to obtain our example.

LEMMA 3.1. There exists a compact Hausdorff space $M$ ond a regular Borel measure $\mu$ on $M$ such that

(1) the carrier of $\mu$ is $M$,

(2) $M$ has no isolated points, and

(3) if $y$ is an essentially bounded $\mu$-measurable function on $M$, then there exists a continuous function $x$ on $M$ such that $x(m)=y(m)$ almost everywhere.

Proof. Let $v$ denote the Lebesgue measure on $[0,1]$. The ring $L_{\infty}(v)$ determines a commutative ring of operators, say $Z$, on $L_{2}(v)$ in the following manner; for $x \in L_{\infty}(v)$ and $y \in L_{2}(v)$, let $\left(B_{x} y\right)(t)=x(t) y(t)$ and $Z=\left\{B_{x}: x \in L_{\infty}(v)\right\} . Z$ is isomorphic and isometric to $L_{\infty}(v)$ and we shall show that $Z$ is its own commutant. To this end suppose $A \in B\left(L_{2}(v)\right)$ and $A B_{x}=B_{x} A$ for all $x \in L_{\infty}(v)$, and let $x_{A}(t)=(A I)(t)$, where $I$ is the identically one function in $L_{2}(v)$. Hence, if $y \in L_{\infty}(v) \subset L_{2}(v)$, then $(A y)(t)=\left(A B_{y} I\right)(t)=\left(B_{y} A I\right)(t)=y(t)(A I)(t)=y(t) x_{A}(t)$. 
Since $L_{\infty}(v)$ is dense in $L_{2}(v)$ it follows that $A$ is the operator that is determined by pointwise multiplication with the function $x_{A}, x_{A} \in L_{\infty}(v)$, and $Z$ is its own commutant.

It follows from Theorem 2.1 and Lemma 2.1 that if $M$ is the maximal ideal space of $Z$ and $\mu$ is the measure on $M$ determined by the functional $F\left(B_{x}\right)$ $=\left(B_{x} I, I\right)=\int_{0}^{1} x(t) d v(t)$, then $M$ and $\mu$ satisfy (1) and (3) of this lemma. That $M$ has no isolated points follows from the fact that $v$ is a continuous measure; i.e., $v$ has no atomic parts.

This completes the proof of Lemma 3.1. Throughout the remainder of this paper, $M$ and $\mu$ will remain fixed in accordance with Lemma 3.1.

Definition 3.1. Let $G$ denote the collection of all functions $g$ from $M$ to the two point group $I_{2}=\{0,1\}$. We define addition in $G$ to be the pointwise addition and the topology of $G$ to be the cross product topology of $I_{2}^{M}$, so that $G$ is a compact topological Abelian group. Let $\rho$ denote the normalized Haar measure on $G$ and $\rho \times \mu$ the cross product measure induced by $\rho$ and $\mu$ on the space $G \times M$. $\mathfrak{D}$ denotes $L_{2}(\rho \times \mu), H$ denotes $L_{2}(\rho)$, and $B(\mathfrak{H})$ (resp. $B(H)$ ) denotes the space of bounded linear operators on $\mathfrak{S}$ (resp. $H$ ).

Definition 3.2. (a) If $\alpha$ is a continuous function on $G \times M$, then the operator $A_{\alpha} \in B(\mathfrak{h})$ is defined by $\left(A_{\alpha} \beta\right)(g, m)=\alpha(g, m) \beta(g, m)$ for $\beta \in \mathfrak{G}$. $\mathbb{C}$ is the collection of all such operators.

(b) If $U$ is a compact-open set in $M$ and $g^{\prime} \in G$ such that $g^{\prime}(m)=0$ for $m \in U$, then the operator $T_{U, g^{\prime}} \in B(\mathfrak{S})$ is defined by $\left(T_{U, g^{\prime}} \beta\right)(g, m)=\beta\left(g+g^{\prime}, m\right)$ for $m \in U$ and $\left(T_{U, g^{\prime}} \beta\right)(g, m)=0$ for $m \notin U$. T is the collection of all such operators.

(c) $K \subset C(M) \subset \mathfrak{S}$ is defined to be the collection of all characteristic functions of the form $\chi_{V \times U}$, where $U$ is a compact-open subset of $M$ and $V$ is a compactopen subset of $G$ defined by $V=\left\{g \in G: g\left(m_{i}\right)=g_{0}\left(m_{i}\right)\right\}$ for $g_{0} \in G$ and $\left\{m_{i}\right\}_{i=1}^{n}$ a finite subset of $M$.

(d) For $m \in M, V_{m}^{0}=\{g \in G: g(m)=0\}, V_{m}^{1}=\{g \in G: g(m)=1\}, H_{m}^{0}=\{a \in H$ : $a(g)=0$ for $\left.g \in V_{m}^{1}\right\}$, and $H_{m}^{1}=\left\{a \in H ; a(g)=0\right.$ for $\left.g \in V_{m}^{0}\right\}$.

(e) $R$ is the smallest norm-closed subring of $B(\mathfrak{H})$ containing $\mathfrak{C} \cup T$. We note since $\mathfrak{C}^{*}=\mathfrak{C}$ and $T^{*}=T$ that $R$ is a symmetric ring.

LEMMA 3.2. The linear subspace generated by $K$ is dense in $C(G \times M)$ under the sup norm topology and is dense in the space $\mathfrak{S}$ under the $L_{2}$ topology.

Proof. It follows from Lemma 2.2 that $M$ has topological basis of opencompact sets. Also, the sets $V=\left\{g \in G: g\left(m_{i}\right)=g_{0}\left(m_{i}\right), i=1, \cdots, n\right\}$ form a basis for $G$. Hence, the sets $V \times U, U$ open-compact in $M$ and $V$ of the above form, constitute a compact-open neighborhood basis for $G \times M$. Thus the linear subspace generated by $K$ is dense in $C(G \times M)$ and, consequently, in $\mathfrak{H}$.

LEMMA 3.3. If $B \in B(\mathfrak{H})$ and $B$ commutes with all the operators in $\mathfrak{C}$, then 
there exists $\beta \in L_{\infty}(\rho \times \mu)$ such that, for $\alpha \in \mathfrak{Y},(B \alpha)(g, m)=\beta(g, m) \alpha(g, m)$ almost everywhere $(\bmod \rho \times \mu)$.

Proof. Let $\beta(g, m)=(B I)(g, m)$, where $I(g, m) \equiv 1$. Hence, if $\alpha \in C(G \times M)$, then $A_{\alpha} \in \mathbb{C}$ and $(B \alpha)(g, m)=\left(B A_{\alpha} I\right)(g, m)=\left(A_{\alpha} B I\right)(g, m)=\alpha(g, m) \beta(g, m)$ almost everywhere. Since the extreme ends of the last chain of equalities holds for $\alpha \in C(G \times M)$, it must hold for $\alpha \in \mathfrak{H}$. Also, if $\alpha=\chi_{V \times U} \in K$, then

$$
\int_{V \times U}|\beta(g, m)|^{2} d \rho \times \mu=|B \alpha|^{2} \leqq|B|^{2}|\alpha|^{2}=|B|^{2}(\rho \times \mu(V \times U))^{2}
$$

so that $\beta \in L_{\infty}(\rho \times \mu)$ and, moreover, its $L_{\infty}$ norm is $|B|$.

THEOREM 3.1. If $B \in B(\mathfrak{S})$ and $B$ commutes with all the operators in $R$, then there exists a continuous function $x_{B}$ on $M$ such that, for $\alpha \in \mathfrak{H},(B \alpha)(g, m)$ $=x_{B}(m) \alpha(g, m)$ almost everywhere $(\bmod \rho \times \mu)$.

Proof. In view of Lemma 3.3, there exists $\beta \in L_{\infty}(\rho \times \mu)$ such that $(B \alpha)(g, m)$ $=\beta(g, m) \alpha(g, m)$. If $y(m)=\int_{G} \beta(g, m) d \rho(g)$, then $y \in L_{\infty}(\mu)$ and, by Lemma 3.1, there exists $x_{B} \in C(M)$ such that $x_{B}(m)=y(m)$ almost everywhere $(\bmod \mu)$. Let $B^{\prime}$ be the operator in $B(\mathfrak{H})$ such that, for $\alpha \in \mathfrak{H},\left(B^{\prime} \alpha\right)(g, m)=x_{B}(m) \alpha(g, m)$. We shall show that if $\alpha \in K$, then $(B \alpha, I)=\left(B^{\prime} \alpha, I\right)$ and, since $K$ generates $\mathfrak{H}$, it will follow that $(B \alpha, I)=\left(B^{\prime} \alpha, I\right)$ for $\alpha \in \mathfrak{S}$. This, in turn, will imply that $\left(B^{\prime} A_{\gamma}^{*} \alpha, I\right)=\left(B A_{\gamma}^{*} \alpha, I\right)$ for $A_{\gamma} \in \mathbb{C}$, and, since $B^{\prime}$ and $B$ commute with all operators in $\mathbb{C},\left(B^{\prime} \alpha, \gamma\right)=(B \alpha, \gamma)$ for $\gamma \in C(G \times M)$; consequently, $\left(B^{\prime} \alpha, \gamma\right)=(B \alpha, \gamma)$ for arbitrary $\alpha, \gamma \in \mathfrak{H}$ and, hence, $B=B^{\prime}$.

In order to prove that $\left(B^{\prime} \alpha, I\right)=(B \alpha, I)$ for $\alpha \in K$, let $\alpha=\chi_{V \times U}$ where $U$ is compact-open in $M$ and $V=\left\{g \in G: g\left(m_{i}\right)=g_{0}\left(m_{i}\right), i=1, \cdots, n\right\}$. Let $\left(a_{i j}, \cdots, a_{n j}\right)$, $j=1, \cdots, 2^{n}$, be all possible $n$-tuples of zeros and ones and set $g_{j}\left(m_{i}\right)=a_{i j}$ and $g_{j}(m)=0$ for $m \neq m_{i}, i=1, \cdots, n$. The collection of sets $\left\{V+g_{j}\right\}_{j=1}^{2^{n}}$ form a pairwise disjoint compact-open cover of $G$.

Now parts (1) and (2) of Lemma 3.1 imply that no point of $M$ has positive $\mu$ measure; hence, for $\delta>0$, there exists a compact open set $U^{\prime} \subset U$ such that $\mu\left(U-U^{\prime}\right)<\delta$ and $m_{i} \notin U^{\prime}$ for $i=1, \cdots, n$.

It follows now (cf. (b) of Definition 3.2) that the operator $T_{U^{\prime}, g_{j}} \in T$ for $j=1, \cdots, 2^{\prime \prime}$; moreover $\left(B T_{U^{\prime}, g_{j}} \alpha, I\right)=\left(T_{U^{\prime}, g_{j}} B \alpha, I\right)$, i.e.,

$$
\begin{aligned}
\int_{M} \int_{G} \beta(g, m) \chi_{V \times U^{\prime}}\left(g+g_{j}, m\right) d \rho(g) d \mu(m) \\
\quad=\int_{M} \int_{G} \beta\left(g+g_{j}, m\right) \chi_{V \times U^{\prime}}\left(g+g_{j}, m\right) d \rho(g) d \mu(m) .
\end{aligned}
$$

Now using the invariance of the Haar measure and the facts $\rho(V)=2^{-n}$ and $-g=g$, we have: 


$$
\begin{aligned}
& \int_{M} \int_{G} \beta(g, m) \chi_{V \times U^{\prime}}(g, m) d \rho(g) d \mu(m) \\
& =2^{-n} \sum_{j=1}^{2^{n}} \int_{M} \int_{G} \beta\left(g+g_{j}, m\right) \chi_{V \times U^{\prime}}\left(g+g_{j}, m\right) d \rho(g) d \mu(m) \\
& =2^{-n} \sum_{j=1}^{2^{n}} \int_{M} \int_{G} \beta(g, m) \chi_{V \times U^{\prime}}\left(g+g_{j}, m\right) d \rho(g) d \mu(m) \\
& =2^{-n} \int_{U^{\prime}} \sum_{j=1}^{2^{n}} \int_{V+g_{j}} \beta(g, m) d \rho(g) d \mu(m)=2^{-1} \int_{U^{\prime}} \int_{G} \beta(g, m) d \rho(g) d \mu(m) \\
& =2^{-n} \int_{U^{\prime}} x_{B}(m) d \mu(m)=\rho(V) \int_{U^{\prime}} x_{B}(m) d \mu(m) \\
& =\int_{M} \int_{G} x_{B}(m) \chi_{V \times U^{\prime}}(m, g) d \rho(g) d \mu(m) .
\end{aligned}
$$

Letting $\delta$ approach zero, this yields

$$
\begin{aligned}
(B \alpha, I) & =\int_{M} \int_{G} \beta(g, m) \chi_{V \times U}(g, m) d \rho(g) d \mu(m) \\
& =\int_{M} \int_{G} x_{B}(m) \chi_{V \times U}(g, m) d \rho(g) d \mu(m)=\left(B^{\prime} \alpha, I\right) .
\end{aligned}
$$

This completes the proof of Theorem 3.1.

COROLlaRY 3.1. If $E$ is the ring of operators $B$ on $\mathfrak{S}$ of the form $(B \alpha)(g, m)$ $=x_{B}(m) \alpha(g, m)$ for $x_{B} \in C(M)$, then $E$ is both the commutant and center of $R$; moreover, $M$ is the maximal ideal space of $E$.

Proof. Clearly $E \subset \mathbb{C} \subset R$ and, on the basis of Theorem 3.1, the commutant of $R$ is $E$. It follows that $E$ is the center of $R$. The mapping $B \leftrightarrow x_{B}$ is an isometry of $E$ onto $C(M)$, so that $M$ is the maximal ideal space of $E$.

Lemma 3.4. Let $S$ be the subset of $\mathfrak{H}$ defined by $S=\left\{A_{\alpha} I: A_{\alpha} \in \mathbb{C}\right\}$. Then

(a) $S$ is dense in $\mathfrak{H}$,

(b) $A S \subset S$ for each $A \in R$, and

(c) each $L_{2}(\rho \times \mu)$ equivalence class of $S$ contains exactly one continuous function.

Proof. (a) follows directly from the definition of $\mathbb{C}$. The set $R_{1}$ of all $A$ in $R$ such that $A S \subset S$ is a norm-closed subring of $R$; moreover, $A_{\alpha} S \subset S$ for $A_{\alpha} \in \mathbb{C}$ and $T_{U, g^{\prime}} S \subset S$ for $T_{U, g^{\prime}} \in T$, hence $C U T \subset R_{1}$ and, hence, $R_{1}=R$. (c) follows from the fact that the carrier of $\rho \times \mu$ is $G \times M$. 
LemMA 3.5. For each $A \in R$ and $m \in M$, there is a unique operator $A_{m} \in B(H)$ such that $(A \alpha)(g, m)=\left(A_{m} \alpha_{m}\right)(g)$, where $\alpha_{m} \in H$ and is the function determined by $\alpha_{m}(g)=\alpha(m, g)$ for $\alpha \in S$; finally, the correspondence $A \rightarrow A_{m}$ is a symmetric and norm-decreasing representation of the ring $R$ onto a ring $R_{m} \subset B(H)$.

Proof. For a fixed $n \in M$, let $R_{1}$ be the set of all operators $A$ in $R$ for which there exists an operator $A_{n} \in B(H)$ such that $(A \alpha)(n, g)=\left(A_{n} \alpha_{n}\right)(g)$ for all $\alpha \in S$. $R_{1}$ is a norm-closed subring of $R$ which contains $\mathbb{C}$ and $T$, consequently, $R_{1}=R$. The set $\left\{\alpha_{n}: \alpha \in S\right\}$ is dense in $H$ and for each $A \in R$ and $\alpha \in S, A \alpha$ defines a unique continuous function in $S$. Hence, $A_{n}$ is uniquely defined by $A$. Finally, it is easily seen that the correspondence $A \rightarrow A_{n}$ is a symmetric representation of $R$ and $|A|=\sup _{n \in M}\left|A_{n}\right|$.

THEOREM 3.2. If, for $A \in R, F(A)=(A I, I), I$ the identically one function on $G \times M$, and $f_{m}(A)=\int_{G}(A I)(g, m) d \rho(g)$, then (a) $F(A)=\int_{M} f_{m}(A) d \mu(m)$ is the Tomita decomposition of $F$ described in Theorem 2.1 , and $(\mathrm{b})$ the correspondence $A \rightarrow A_{m}$ of Lemma 3.5, is the representation of $A$ induced by $f_{m}$.

Proof. $F(A)=(A I, I)=\int_{M} \int_{G}(A I)(g, m) d \rho(g) d \mu(m)=\int_{M} f_{m}(A) d \mu(m)$; also, for $B \in E, f_{m}(B A)=\int_{G} x_{B}(m)(A I)(g, m) d \rho(g)=x_{B}(m) f_{m}(A)$, and $f_{m}(A)$ is a continuous function of $m$ for fixed $A$. Hence, in accordance with (1), (2) and (3) of Theorem 2.1, $F(A)=\int_{M} f_{m}(A) d \mu(m)$ is the Tomita decomposition of $F$. Now, from Lemma 3.5, $f_{m}(A)=\int_{G}(A I)(g, m) d \rho(g)=\int_{G}\left(A_{m} I_{m}\right)(g) d \rho(g)=\left(A_{m} I_{m}, I_{m}\right)$ in $H$. This equality, along with the fact that $\left\{A_{m} I_{m}: A \in R\right\}$ is dense in $H$, uniquely defines the representation generated by $f_{m}$ to within unitary equivalence. Hence, $A \rightarrow A_{m}$ is this representation.

THEOREM 3.3. No one of the functionals $f_{m}$, described in Theorem 3.2, is indecomposable and no one of the rings $R_{m}$ is irreducible.

Proof. We shall prove the theorem by showing that the spaces $H_{m}^{0}$ and $H_{m}^{1}$ are invariant under the ring $R_{m}$. To this end, let $R_{m}^{0}$ denote the norm-closed ring consisting of all operators $A_{m} \in R_{m}$ such that $A_{m} H_{m}^{0} \subset H_{m}^{0}$ and $A_{m} H_{m}^{1} \subset H_{m}^{1}$ (cf.(d) of Definition 3.2). Clearly, $\mathfrak{C}_{m}=\left\{A_{m}: A \in \mathbb{C}\right\} \subset R_{m}^{0}$. Let $T_{m}=\left\{A_{m}: A \in T\right\}$. If $T_{U, g^{\prime}} \in T$, then $g^{\prime}(m)=0$ for $m \in U,\left(T_{U, g^{\prime}} \alpha\right)(g, m)=\alpha\left(g+g^{\prime}, m\right)$ for $m \in U$, and $\left(T_{U, g^{\prime}} \alpha\right)(g, m)=0$ for $m \notin U$. Hence, if $\alpha \in S$ and $\alpha_{m} \in H_{m}^{0}$, then $\left(T_{U, g^{\prime}} \alpha\right)(g, m)=0$ for $m \notin U$ and $g \in V_{m}^{1}$. Hence, $A_{m} \alpha_{m} \in H_{m}^{0}$ for $\alpha_{m} \in H_{m}^{0}$ and $A \in T$. A similar argument shows that the same is true for the space $H_{m}^{1}$. Since $\widetilde{C}_{m} \cup T_{m}$ generates $R_{m}$ and $\mathfrak{C}_{m} \cup T_{m} \subset R_{m}^{0} \subset R_{m}$, we have $R_{m}=R_{m}^{0}$ and, consequently, $H_{m}^{0}$ and $H_{m}^{1}$ are invariant subspaces of $R_{m}$. Hence, no $R_{m}$ is irreducible and no $f_{m}$ is indecomposable.

This concludes the proof of Theorem 3.3; however, a few observations about the functional $f_{m}$ may be worthwhile. For each $m \in M$, the functional $f_{m}$ can be written as $f_{m}^{0}+f_{m}^{1}$ where 


$$
f_{m}^{0}(A)=\int_{V_{m}^{0}}\left(A_{m} I_{m}\right)(g) d \rho \text { and } f_{m}^{1}(A)=\int_{V_{. .}^{1}}\left(A_{m} I_{m}\right)(g) d \rho, A \in R .
$$

The functionals $f_{m}^{0}$ and $f_{m}^{1}$ are distinct positive functionals on $R$ having the interesting property that $\int_{M} f_{m}^{0}(A) d \mu=\int_{M} f_{m}^{1}(A) d \mu=2^{-1} \int_{M} f_{m}(A) d \mu$ for $A \in R$. This emphasizes one essential drawback of the Tomita decomposition, namely, unless $R$ is weakly closed, the rings $R_{m}$ may have important properties individually which are not reflected in the behavior of $R$ as a ring of operators in $\mathfrak{I}$.

IV. Remark. Since the publication of Tomita's paper, several papers have appeared on the more general problem of expressing a point in a convex-compact set $K$ as a direct integral over the set $N$ of extremal points of $K$. Choquet [2], [3], Bishop and deLeeuw [1], and Loomis [4] have all obtained results on this problem. Referring to Loomis's paper, we note that these results can be applied to the Tomita problem when $K$ is the set of normalized positive functionals on a ring $R$ which contains its commutant $E$. The results of Loomis yield a unique measure $\mu$ on $K$ having the properties (1) $F(A)=\int_{K} f(A) d \mu(f)$, and (2) $\mu$ is zero on any $G_{\delta}$ set which misses $N$. The carrier of $\mu$ is homeomorphic to $M$; however, Theorem 3.3 shows that the carrier of $\mu$ may be disjoint from $N$ in spite of (2).

It is possible to use the measure $\mu$ to define a measure $\omega$ on the set algebra consisting of all sets of the form $N \cap \Delta$ where $\Delta$ is a Baire set of $K$. With a slight modification of the definition of direct integrals, $\omega$ yields a direct integral decomposition of $R$ into irreducible rings $R_{f}$. Unfortunately $\omega$ is not a regular Borel measure on a compact space, so that some of the important properties of a direct integral are lost.

\section{BIBLIOGRA PHY}

1. E. Bishop and $\mathrm{K}$. deLeeuw, The representation of linear functionals by measures on sets of extreme points, Ann. Inst. Fourier (Grenoble) 9 (1959), 305-331.

2. G. Choquet, Unicité des représentations intégrales au moyen de points extrémaux dans les cônes convexes réticulés, C. R. Acad. Sci. Paris 243 (1956), 555-557.

3. - Existence des représentations intégrales au moyen de points extrémaux dans les cônes convexes, C. R. Acad. Sci. Paris. 243 (1956), 699-702.

4. L. H. Loomis, Unique direct integral decomposition on convex sets, Amer. J. Math. 84 (1962), 509-526.

5. M. A. Naimark, Normed rings, Noordoff, Groningen, 1959.

6. M. Tomita, Representations of operator algebras, Math. J. Okayama Univ. 3 (1954), 147-173.

Louisiana State University,

Baton Rouge, Louisiana 